\title{
On the Molecular Structure of Circumstellar Envelopes Surrounding C Stars
}

\author{
A. BEATE C. PATZER, JAN MARTIN WINTERS \\ and ERWIN SEDLMAYR
}

Institut für Astronomie und Astrophysik, TU Berlin, Germany

Circumstellar envelopes around cool carbon stars show a rich chemistry as indicated by observations of many different molecular species. Most of the molecules are detected in the shell of the nearby C star IRC +10216. In recent years detailed information on the spatial distribution of molecules has been obtained from high-resolution interferometry and spectroscopy. Within circumstellar envelopes, widely different physical conditions are encountered: high densities and high temperatures prevailing in the inner shell, and cool rarefied conditions predominating in the outer part, which is exposed to the interstellar radiation field.

Based upon the model structure of a stationary dust-driven wind we have investigated the molecular structure of the entire circumstellar shell. The chemical composition of the envelope has been determined by solving a network of chemical rate equations including gas kinetic reactions and photochemical processes. Taking dust shielding and the wavelength dependence of the photo-reactions explicitly into account, the photo rates have been calculated by integration of the mean intensity of the local radiation field over the associated frequency-dependent photo cross section.

Starting from the photosphere with chemical equilibrium abundances as the initial condition, characteristic features of the molecular structure of the circumstellar envelope are reproduced by the model. In general the theoretical molecular distributions tend to be less extended than the observed ones. Possible reasons are an overestimation of the interstellar radiation field and the neglect of molecular shielding effects. Therefore, further investigations will account for these effects.

This work was supported by the BMBF grant 05 3BT 13A 6 . 